\title{
Development of 3D Printed Shoe Designs Using Traditional Muntin Patterns
}

\author{
Ho Sun Lim† \\ Dept. of Clothing \& Textiles, Sookmyung Women's University; Seoul, Korea
}

\begin{abstract}
This study proposes 3D printed shoe designs with patterns made by reinterpreting traditional Korean muntin patterns as customized designs that are unique to individual consumers and different from existing products. In the fashion industry, shoes with diverse designs grafted with 3D printing technology have been introduced. Artistic 3D printed shoes showcase the unique designs of designers. Functional and practical 3D printed shoes that can be worn during daily activities and during exercise have been actively developed. Traditional Korean pattern designs are also being recreated into designs reflecting the aesthetic sense of modern times with our own identity. The uniqueness of the traditional muntin patterns in geometric shapes, such as intersections of lines, rectangles, and octagons, are expressed in shoe designs with modern aesthetic senses by utilizing the traditional patterns that conform well to the modern geometric beauty of forms. This study was intended to develop 3D shoe designs that reinterpreted the motif of muntin patterns from among traditional Korean geometric patterns with a modern aesthetic sense. The octagonal patterns that express the scenery of spring can be seen through the muntins in traditional Korean-style houses were designed on the heels of shoes. Utilizing the Rhino CAD program and ProJet 660 Pro 3D printer, shoes were designed and printed. The processes for making shoes using 3D printing technology proposed in this study are significant because they represent the creation of designs in a new area. The results of this study might help in the development of 3D printed fashion products.
\end{abstract}

Key words : 3D printed shoes, traditional pattern, design process, 3D CAD design

\section{Introduction}

The increasing importance of cultural diversity and the unique traditional cultures of various countries have led to efforts to develop cultural products reflecting the traditional Korean beauty and aesthetic sense. Since modern consumers are more likely to prefer products that possess a unique individuality, differentiated apparel products made modern by reconstructing traditional Korean designs should be developed (Jung et al., 2014). That is, designs should be developed that not only reflect traditional culture but also grafted with modern aesthetic senses and technologies in response to customer needs.

Muntin patterns are one of the traditional patterns widely utilized to emphasize Korean emotions, and the designs show the beautiful formative aesthetic that reflects the daily life of our ancestors and their artistry (Hong \& Kim, 2011; Kim, 2016).

Muntin patterns form numerous geometric shapes with vertical, horizontal, and diagonal lines intersecting each other. The charac-

$\dagger$ Corresponding author; Ho Sun Lim

Tel. +82-2-2077-7121, Fax. +82-2-2077-7324

E-mail: lhs@sm.ac.kr

(C) 2017 (by) the authors. This article is an open access article distributed under the terms and conditions of the Creative Commons Attribution license (http://creativecommons.org/licenses/by/3.0/), which permits unrestricted use, distribution, and reproduction in any medium, provided the original work is properly cited. teristics of muntin patterns include the harmony between the lines of muntins and the faces in window paper, and the geometric shapes such as triangles, rectangles, hexagons, and octagons composed of many lines. In addition, the subdued light that passes through the window paper and the shadows of the muntins express more diverse geometric shapes (Kang \& Kim, 2008).

Therefore, the uniqueness of the traditional patterns in geometric shapes, such as intersections of lines, rectangles, and octagons, are expressed in shoe designs with modern aesthetic senses by utilizing the traditional patterns that conform well to the modern geometric beauty of forms.

As the personal preferences of modern consumers diversify, the demand for personalized products that are unique for each individual customer has been increasing. Thanks to the recent development of information and communications technology, production systems that can manufacture customized products are changing. Shoe manufacturers such as Nike and Adidas have recognized these changes and now offer customized shoe services. In addition, they are focusing on the development of high-performance sneakers utilizing 3D printing technology.

Recently, 3D printing technology that enables the creation of products with complex shapes and innovative designs by stacking many layers of raw materials one by one has been applied in diverse industrial fields, while creating new paradigms of manufacturing technologies (Byun, 2016; Lee \& Lee, 2016). In the field 
of fashion, designers are creating innovative and formative fashion designs under the concept termed as the convergence of fashion and 3D printing technology (Kim et al., 2015). As such, 3D printing technology enables small batch production. Therefore, customized shoes with designs unique to individual consumers can be efficiently fabricated.

This study proposes 3D printed shoe designs with patterns made by reinterpreting traditional Korean muntin patterns as customized designs that are unique to individual consumers and different from existing products. This study is relevant in that it develops creative designs in a new field by presenting $3 \mathrm{D}$ printed shoe design processes that reflect the convergence of digital technologies and traditional fashion design.

\section{Theoretical Background}

\subsection{Traditional pattern design}

Patterns are defined as the configuration of points, lines, and/or colors into figures to stimulate aesthetic senses. Traditional Korean patterns are patterns and forms that reflect our ancestors' excellent creativity, knowledge, artistry, and life based on our unique historical background ("Korean Culture Portal”, 2016). Traditional Korean pattern designs are also being recreated into designs reflecting the aesthetic sense of modern times with our own identity. Traditional Korean patterns can be divided into human patterns, animal patterns, plant patterns, artifact patterns, natural scenery patterns, character patterns, geometric patterns, and complex patterns, according to their shapes and materials ("Korean Culture Portal", 2016; Ye et al., 2013). Geometric patterns are the most frequently used among the traditional patterns used to express Korean emotions. These geometric patterns include letters, dancheong, muntin, rice cake patterns, roof-end tiles, and taegeuk (Hong \& Kim, 2011).

Among the many traditional patterns, muntin patterns form shapes that have the conciseness of geometric structures and reflect the traditional formative aesthetic through the composition of faces created by intersections of lines. According to compositions of intersections of muntin lines, traditional muntin patterns can be divided into those composed of vertical lines, vertical and horizontal lines, diagonal lines, diagonal and vertical lines; diagonal, vertical, and horizontal lines; patterns made by simplifying the shapes of letters or animals, and those made by shaping flower patterns (Kang \& Kim, 2008; Kim, 2016).

Examples of products that utilize traditional Korean patterns include the "An-Chae" line of kitchen furniture (Fig. 1) released by Enex in 2007. The furniture expressed Korean beauty such as handles inspired by the muntin of traditional Korean houses and the doorknobs of traditional doors with paper windows. “休 \& Funny”

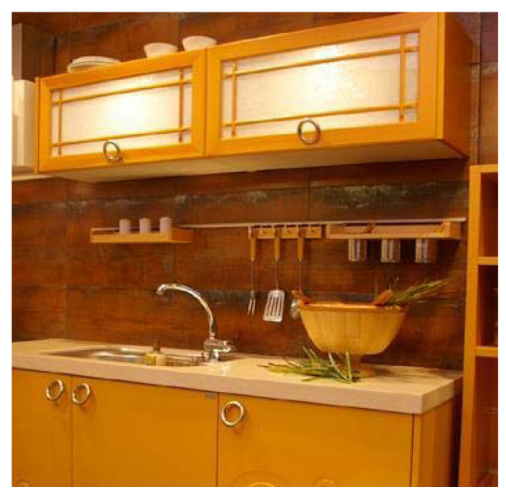

Fig. 1. Ennex 'An-Chae'. http://www.edaily.co.kr

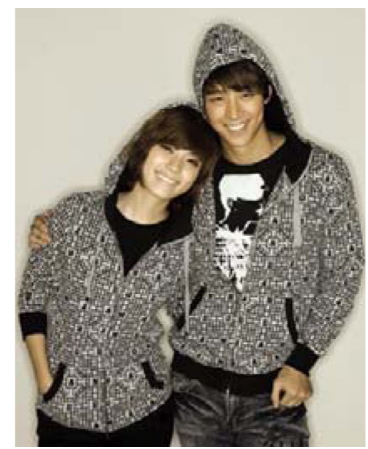

Fig. 2. Jambangee traditional patterned T-shirt. http://news.inews24.com

featured the convergence of kitchen furniture that emphasized traditional beauty ("Enex released kitchen furniture", 2007). In 2009, the denim brand Jambangee released T-shirts that featured traditional Korean patterns such as the traditional door with paper windows and muntin patterns as motifs (Hong, 2009).

\subsection{Current state of $3 \mathrm{D}$ printed fashion product development}

Shoes are traditionally made using lasts, which are models of feet made by fabricating frames of molds and then pouring plastic substances into the frames. The leather or fabric is then cut according to the design, followed by gluing and sewing processes. In contrast, when utilizing 3D printing technology, shoes can be produced instantaneously without fabricating the frames of molds, thus reducing the steps in the production process and allowing the implementation of complex and characterful designs.

3D printing techniques can be classified into three types: fused deposition modeling, which melts and creates stacks of solid filaments; stereo lithography apparatus, which cures liquids by shooting laser beams; and selective laser sintering, which melts powderbased materials with high-intensity laser beams to create sculptures (Song \& Kim, 2015). In the fashion industry, shoes with diverse 


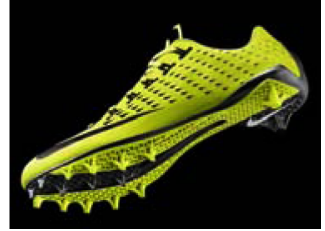

Fig. 3. Nike shoes. https://www.fool.com

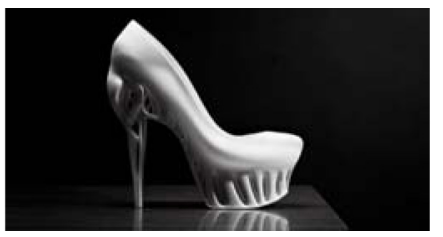

Fig. 6. Marieka Ratsma's shoes. http://trendland.com

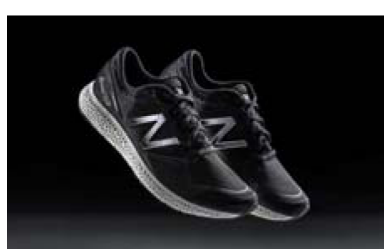

Fig. 4. New Balance shoes. https://www.fool.com

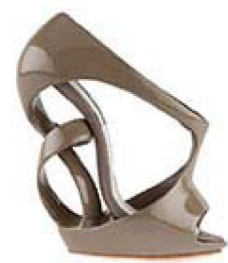

Fig. 7. Victoria Spruce's shoes. http://www.additivefashion.com

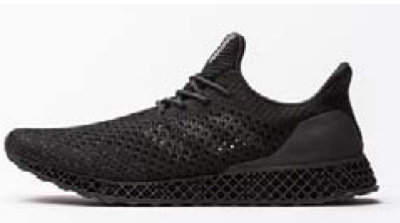

Fig. 5. Adidas shoes. http://news.adidas.com

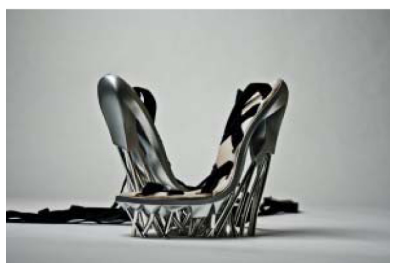

Fig. 8. Brian Oknyansky's shoes. https://all3dp.com designs grafted with 3D printing technology have been introduced. Artistic 3D printed shoes showcase the unique designs of designers. Functional and practical 3D printed shoes that can be worn for daily activities and during exercise have also been actively developed (Byun, 2016; Yang, 2013).

Sports shoes such as Nike's soccer shoes (Fig. 3), New Balance's high-performance running shoes (Fig. 4), and Adidas's 3D runner (Fig. 5) were produced using 3D printing technology. Designer Marieka Ratsma designed 3D printed shoes (Fig. 6) inspired by the bird's cranium, while designer Victoria Spruce designed 3D printed shoes (Fig. 7) inspired by organic ceramic sculptures with continuous and never-ending lines. Designer Brian Oknyansky designed a 3D printed shoe (Fig. 8) featuring uniquely shaped spiral heels made of titanium.

In addition, studies have been actively conducted on 3D printed shoe design, including shoe design, heel design, and customized

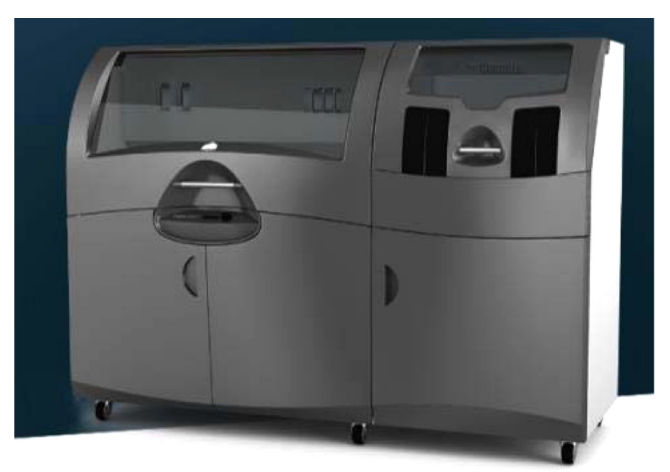

Fig. 9. Projet 660 Pro of 3D Systems Co. https://www.3dsystems.com insole design (Byun, 2016; Kim \& Jung, 2015; Lee \& Lee, 2016).

\section{Methods}

This study was aimed at developing 3D shoe designs that reinterpreted muntin patterns from among traditional Korean geometric patterns with modern aesthetic senses. To express Korean sentiments, muntin patterns were grafted onto modern shoes as a design concept in order to design octagonal shoe heels with geometric muntin patterns.

Using the 3D CAD program Rhinoceros 5, muntin patterns from traditional Korean-style houses were sketched and designed. Thereafter, prototypes of 3D printed shoes were made using VisiJet PXL (powder material) and ProJet 660 Pro (Fig. 9), a 3D printer for ColorJet Printing 3D Systems.

\section{Results}

\subsection{Traditional patterned 3D design}

In this study, traditional muntin patterns from traditional Koreanstyle houses (Fig. 10(a)) were selected as the main motif. The traditional muntin patterns were developed by reconstructing the motif with modern techniques using the Rhinoceros 5 program. The design concept was a "spring landscape" expressed through the muntins. The intersecting horizontal and vertical lines of the muntins were combined with the formative beauty of circles and octagons to reconstruct the muntins into repetitive and organic forms, thereby spreading the muntins. That is, petals were created through the intersections of the horizontal and vertical lines in 


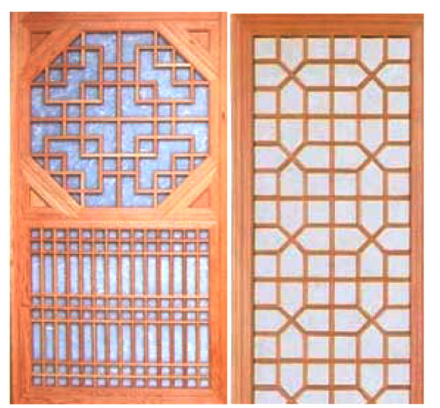

(a)

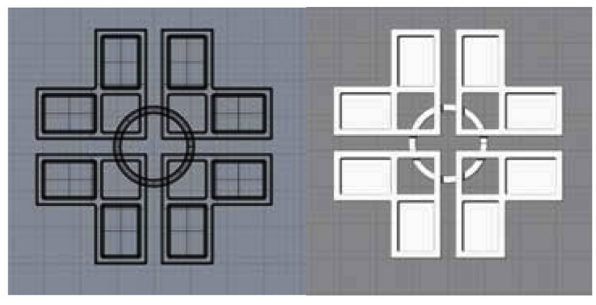

(b)

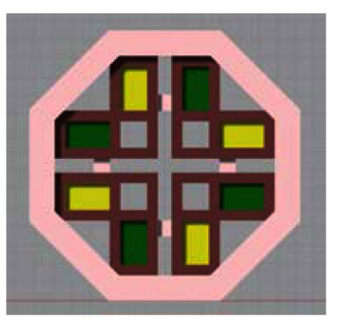

(c)

Fig. 10. Development of traditional muntin pattern designs; (a) traditional muntins from traditional Korean-style houses. http://blog.naver.com, (b) development of muntin pattern designs, (c) completion of octagonal muntin pattern designs.

octagonal borders; the petals were rotated 360 degrees to represent fully blooming flowers (Fig. 10(b)). In addition, the pink color of blooming flowers and the green color of grass and leaves were used together with the brown color of traditional muntins (Fig. $10(\mathrm{c}))$.

\subsection{D CAD shoes design}

The traditional muntin patterns of the "spring landscape" inspired by the muntins of traditional Korean-style houses were applied to the heels and vamps of shoes. Unique and complex designs can be expressed through 3D printing since this method produces shoes by laminating the material, unlike existing methods that require metal molds and liquid phase materials, such as plastic, to be poured into the molds. In other words, this study was intended to express traditional Korean muntin designs with unique and for-

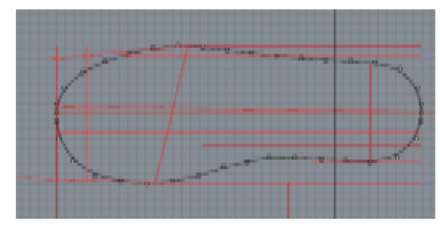

(a)
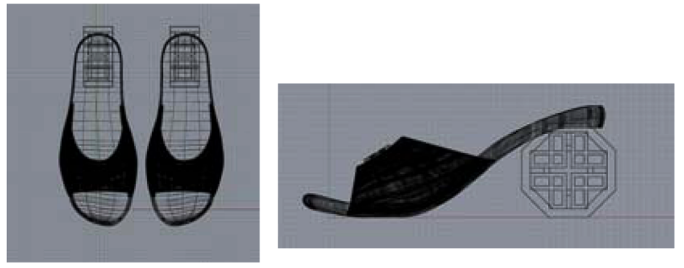

(c) mative characteristics that cannot be reproduced through conventional manufacturing processes on the heels of shoes. These designs not only play functional roles but also show aesthetic effects. The bottom and upper parts of shoes were designed using the Rhinoceros 5 CAD program. First, reference lines for the design of $235 \mathrm{~mm}$ shoes were drawn with polylines and the contours of the outsoles were designed (Fig. 11(a)). Thereafter, heels with traditional muntin patterns were designed with an octagonal shape measuring $7.2 \mathrm{~cm}$ (height) $\times 7.2 \mathrm{~cm}$ (width) $\times 3.5 \mathrm{~cm}$ (depth), and the outsole was adjusted to fit the heels (Fig. 11(b)). The vamps of the uppers that cover the foot were designed on the outsoles (Fig. 11(c)). In the final stage, layers were made for the shoe part and colors were designated for the vamp area of the upper and outsole areas (Fig. 11(d)). The prototype shoe designs were stored in 3DM file format.
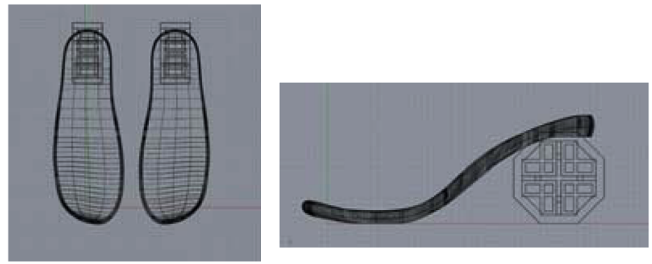

(b)
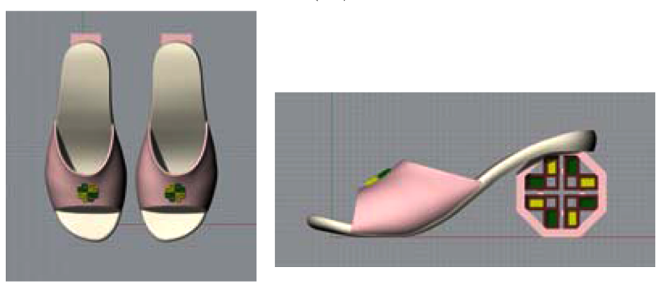

(d)

Fig. 11. Process of shoe designing utilizing the Rhinoceros 5 program; (a) Design of the reference line and contour of the outsole of shoes, (b) Design of shoe soles and heels, (c) Design of shoe uppers, (d) Completion of color-designated shoe designs. 


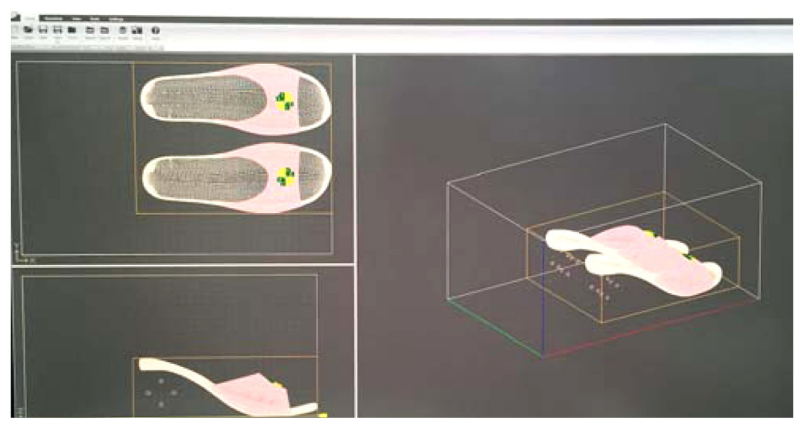

Fig. 12. 3D printer output program of 3D Systems Co.

\subsection{D printed shoes production}

To produce the shoes in the $3 \mathrm{D}$ printer, the shoe design file in $.3 \mathrm{dm}$ format was imported from the 3D Systems printer output program (Fig. 12). The setting window for 3D printing output was activated to set the output precision and layer quantity. Fig. 13(a) shows the laminated cross-sectional layers made for the output of a $3 \mathrm{D}$ printer. Thereafter, when the output passed through the $3 \mathrm{D}$ printer, the binder is sprayed through the inkjet head to bond the powder materials, and the power material was laminated from the bottom of the shoes upward, while being colored to produce the 3D printed shoes (Fig. 13(b)). When 3D printing was completed, the powder on the shoes was completely removed using airbrushes (Fig. 13(c)). Finally, the shoes were immersed in colored liquid and then dried (Fig. 13(d)). Fig. 14 shows the 3D printed shoes with traditional muntin patterns produced from the abovementioned process.
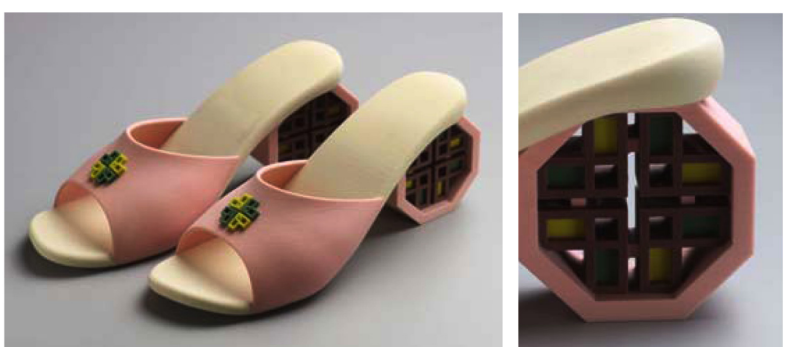

Fig. 14. 3D printed shoes made utilizing traditional muntin patterns.

\section{Conclusion}

3D printing technology has received significant attention globally and its application in diverse fields demonstrate its influence. In the field of fashion, 3D printing is applied to innovative designs as a new aesthetic concept referred to as the convergence of digital technology and design. In this study, designs that reinterpreted traditional Korean beauty with modern aesthetic senses were developed, and 3D printed shoes were created utilizing 3D CAD and 3D printing technologies. First, octagonal patterns that expressed spring scenery seen through the muntins in traditional Korean-style houses were designed with the Rhinoceros 5 CAD program. Second, the designed patterns were applied to the heels and the vamp parts of the uppers of shoes. Then, the outsole and vamp of the upper part of the shoes were designed. Third, 3D printed shoes were generated with the ProJet 660 Pro 3D printer. The shoes were colored using liquid coloring materials.
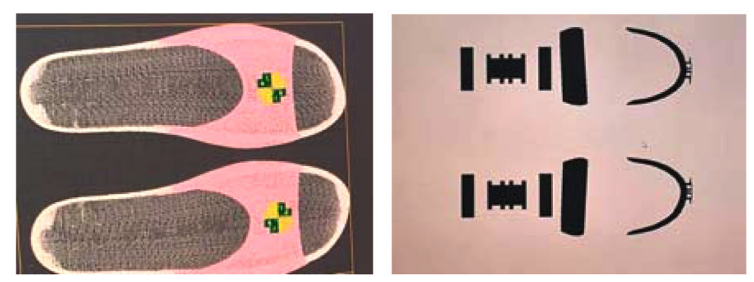

(a)

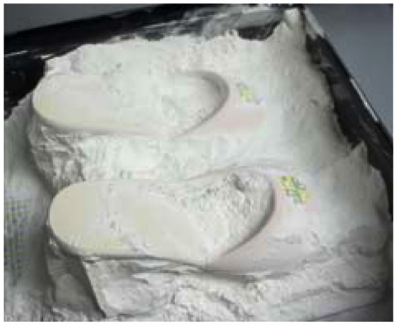

(c)

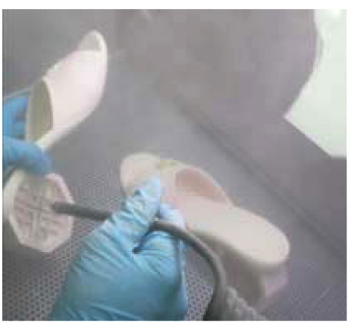

(d)

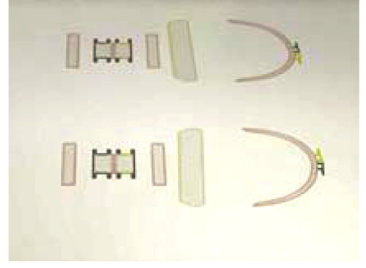

(b)

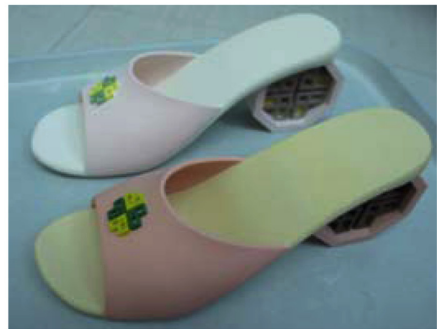

(e)

Fig. 13. 3D printed shoes production process; (a) Cross-section layers of 3D printed shoes, (b) output process, (c) output completion, (d) power removal process, (e) coloring process. 
This study is relevant in that it redesigned traditional patterns to make them suitable for the expression of Korean traditional images in products with modern aesthetic senses as well as for shoe designs applied with 3D printing technology.

In addition, this study proposed shoe heel designs unique to individual consumers, reflecting the increasing consumer demand for customized designs. Shoes can be fabricated utilizing 3D printing technology that is efficient for small batch production. This study also showed the possibility expressing new aesthetics for innovative and formative designs in the field of fashion by proposing shoe design processes with $3 \mathrm{D}$ printing technology that can generate innovative and complex designs. However, one limitation of this study is the difficulty of ensuring the wearability of products owing to restrictions in type of $3 \mathrm{D}$ printing materials that can be used to manufacture these products. If new 3D printing materials and software for fashion products are developed in the future, products with high marketability can be actively developed.

\section{Acknowledgement}

This research was supported by the Sookmyung Women's University research grants (Project No. 1-1503-0189).

\section{References}

3D Systems. (2017). Project CJP 660Pro. Retrieved February 23, 2017, from https://www.3dsystems.com/3d-printers/projet-cjp-660pro

'Adidas shoes'. (2016, December 15). Adidas' News Stream. Retrieved February 6, 2017, from http://news.adidas.com/us/Latest-News/ adidas-makes-first-3d-shoe-available-for-purchase-with-exclusivedrop/s/7004e8fa-324b-4b71-bc2f-aacf3d61b135

'Brian Oknyansky's shoes'. (2015, February 21). 3D printed shoes. All About 3D Printing. Retrieved January 30, 2017, from https:// all3dp.com/3d-printed-womens-shoes/

Byun, H. J. (2016). A study on the relation between 3D printing technology and shoes design changes -Focused on technological background and present situation-. Journal of Formative Media Studies, 19(4), 101-112.

'Enex released kitchen furniture 'An-Chae' containing Korean beauty'. (2007, February 27). Edaily. Retrieved November 11, 2016, from http:/ /www.edaily.co.kr/news/NewsRead.edy?SCD =JC41\&newsid $=02151686583034784 \& D C D=A 00304 \&$ OutLnkChk $=Y$

Hong, J. H., \& Kim, H. K. (2011). The development of woman's daily hanbok textile design appling Korea traditional flower pattern. Fashion \& Textile Research Journal, 13(6), 848-855.

'Jambangee traditional patterned T-shirt'. (2009, September 29). Denim brand Jambangee released 'traditional pattern' series. inews24. Retrieved August 20, 2016, from http://news.inews24. $\mathrm{com} / \mathrm{php} /$ news_view.php?g_menu $=703110 \&$ g_serial $=446038$

Jung, D. W., Kim, T. E., Bang, H. E., Cho, S. H., \& Kim, M. J. (2014).
The cultural identity found in tote bags as a cultural product and the a development of a design -Focused on hanbok motifs-. Journal of the Korean Society of Clothing and Textiles, 38(4), 506517. doi:10.5850/JKSCT.2014.38.4.506

Kang, K. A., \& Kim, S. M. (2008). A study on formativeness of geometric shapes on latticework made in the era of Joseon dynasty. Journal of the Korean Society of Design Culture, 14(3), 1-11.

Kim, J. H., \& Jung, E. C. (2015). Modeling process development for customized woman midsole design based-on 3D printing technology -Focused on a women in her twenties with 235240mm foot size-. Journal of Korean Design Forum, 46, 267-277.

Kim, K. T. (2016). A study on jewelry design parts with the application of traditional patterns -Traditional window patterns as the motif-. Design Convergence Study, 15(5), 213-225.

Kim, Y. S., Lee, J. A., Kim, J. H., \& Jun, Y. S. (2015). Formative characteristics of $3 \mathrm{D}$ printing fashion from the perspective of mechanic aesthetic. The Research Journal of the Costume Culture, 23(2), 294-309. doi:10.7741/rjcc.2015.23.2.294

Korean Culture Portal (2016). Understanding of patterns. Retrieved December 20, 2016, from http://www.culture.go.kr/tradition/ pattern.do

'Korean tradition'. (2010, September 12). Retrieved March 6, 2016, from http://blog.naver.com/hwarm/80115243835

Lee, J. S., \& Lee, J. J. (2016). A study on the development of shoe design using 3D scanning and 3D printing -Focused on heel design-. Journal of the Korean Society of Fashion Design, 16(2), 99-111. doi:10.18652/2016.16.2.7

'Marieka Ratsma's shoes'. (2012, November 12). Marieka ratsma: Biomimicry shoe. Trendland. Retrieved February 9, 2017, from http://trendland.com/marieka-ratsma-biomimicry-shoe/

'New Balance shoes.' (2016, April 2). 3D-printed shoe race: How do Nike, Under armour, and Adidas stack up?. The Motley Fool. Retrieved December 22, 2016, from https://www.fool.com/ investing/general/2016/04/02/3d-printed-shoes-nike-underarmoradidas-newbalance.aspx

'Nike shoes'. (2016, April 2). 3D-printed shoe race: How do Nike, Under armour, and Adidas stack up?. The Motley Fool. Retrieved December 22, 2016, from https://www.fool.com/investing/general/ 2016/04/02/3d-printed-shoes-nike-underarmor-adidas-new balance.aspx

Song, H. W., \& Kim, S. H. (2015). A third industrial revolution 3D printing. KIC News, 18(1), 27-44.

'Victoria Spruce's shoes'. (2013, April 27). Shoes by Victoria Spruce. Additive Fashion. Retrieved February 3, 2017, from http:// www.additivefashion.com/shoes-by-victoria-spruce/

Yang, J. W. (2013). A study on the fashion design adapting threedimensional digital printing technology. Unpublished master's thesis, Hongik University, Seoul.

Ye, I. G., Kim, D. U., \& Chung, I. H. (2013). Modernization of the Korean traditional patterns and its application to t-shirts. Journal of the Korean Society of Clothing and Textiles, 37(3), 207-279. doi:10.5850/JKSCT.2013.37.3.270

(Received 16 March 2017; 1st Revised 2 April 2017; 2nd Revised 26 April 2017; Accepted 28 April 2017) 